respectively, $Z=2.266, p=0.023)$ and at night $(4.3 \pm 6.12 \mathrm{pg} /$ $\mathrm{ml}$ vs $13.79 \pm 5,931 \mathrm{pg} / \mathrm{ml}$, respectively, $Z=2.888, \mathrm{p}=0.004)$ in obese patients. Morning and day melatonin levels in both groups showed no significant differences, but there is a trend towards its increasing in obese adolescents in the morning hours $(4.73 \pm 3.23 \mathrm{pg} / \mathrm{ml}$ in main group and $4.32 \pm 2.35 \mathrm{pg} / \mathrm{ml}$ in control group, $\mathrm{Z}=0.044, \mathrm{p}=0.965$ ).

Conclusions The results of this study indicate a significant decrease in the melatonin levels and disturbance of the melatonin production rhythm. In obese adolescents, we revealed a trend towards melatonin secretion shift to the early morning, when the melatonin level must decrease in healthy adolescents. Because of the varied roles of melatonin in human metabolic rhythms (insulin, leptin, adiponectin secretion and other biological active substances), these results suggest that to return to a normal rhythmicity and to lose weight, melatonin administration in evening hours and morning bright light therapy are reasonable and necessary.

\section{OC18 A NOVEL STANDARDISED PROTOCOL FOR POST- OPERATIVE ANTIBIOTIC USE IN APPENDICITS. RBHSC STUDY RESULTS AT SIX MONTHS}

David Colvin*, Evelyn Ervine, Paul Moriarty, Alistair Dick. Royal Belfast Hospital for Sick Children, Belfast, UK

\subsection{6/archdischild-2019-epa.18}

Background Variation persists nationwide in the post-operative management of complicated appendicitis. Following a review of recent publications, it seems that the most beneficial change to patient outcome is the use of a standardised departmental approach. This has been shown to be effective in multiple centres using clinical findings and white cell count (WCC). A local review however suggested that in our patient population, post-operative complications were more strongly correlated with elevated C-reactive protein (CRP).

We implemented a novel protocol using CRP with clinical findings to standardise our post-operative antibiotic use and here present our findings after six months

Methods A prospective data collection was completed to assess:

Complicated appendicitis rate (operator observed perforated or gangrenous appendix)

Post-operative length of stay

Infective complication rate (Abscess or wound infection of Clavien Dindo Grade 2+)

This was compared to all patients undergoing an appendicectomy over the previous six months.

Results 42 patients completed the new protocol over six months (New), with 53 in the six months prior (Prior).

New vs Prior; Age range was similar: 9 (6-12 IQR) vs 9 (7-11) and normal appendicectomy rate improved $4.7 \%$ (2/ 42) vs $11.3 \%(6 / 53)$.

Complicated appendicitis rate was $50 \%$ (21/42) vs $42 \%$ (22/53).

Mean post-operative length of stay for the complicated group did not change, 5.6 vs 5.9 days $(\mathrm{p}=0.38)$, and improved in the simple group, 2.1 v 3.1 days $(p=0.01)$.

Infective complication rate improved on the new protocol $9 \%(2 / 22)$ vs $27 \%(6 / 22)$.

Conclusions After six months with the New protocol the postoperative length of stay did not increase for patients with complicated appendicitis and improved for those with simple appendicitis.

Significantly the rate of post-operative infective complications has fallen.

Our group hypothesise that the use of a standardised approach provides parents and staff with a clear post-operative plan and expedites discharge for patients with simple appendicitis. It also rationalises blood sampling and the antibiotic regimen appears to reduce infective complications.

We plan to continue using the protocol and will review our results at one year.

\section{OC19 CHANGING THE SAFETY PARADIGM - BRINGING SITUATION AWARENESS TO THE FORE}

1,2Peter Lachman*, ${ }^{1}$ Kasia Muszynska. ${ }^{1}$ The Royal College of Paediatrics and Child Health,
London, UK: ${ }^{2}$ The International Society for Quality in Healthcare (ISQua), Dublin, Ireland

\subsection{6/archdischild-2019-epa.19}

Context Situation Awareness for Everyone (SAFE) is a patient safety programme developed by RCPCH in London as part of a Health Foundation Initiative Closing the Gap in Patients Safety. The evolution of the programme was to take complex patient safety theory and methodology and translate it into day to day clinical practice.

Problem Deterioration of children is a major problem worldwide, in all contexts. Patient safety has yet to find ways to protect children from missed or delayed diagnosis and this programme aimed at developing proactive anticipation of deterioration to prevent adverse outcomes. This is against a background of variable levels of safety culture and a belief system that may not facilitate continual improvement.

Intervention This programme was run in four waves; in 2014-2016 three waves were held involving 28 clinical teams across England including all the leading children's hospitals. The final wave was held in 2017 with a further 23 hospitals. Over the course of the programme we developed new ways of bringing the patient safety concepts to the frontline staff. The actual intervention was a structured safety huddle which was held regularly in different settings. The aim was to bring situation awareness or a shared understanding of clinical conditions of children for staff. This approach integrated risk management, human factors, resilient engineering and reliability theory within a person focussed framework, while implementing multidisciplinary team huddles, which allowed participants to learn how to predict the changes in clinical status of children, rather than react to them.

Measurement of improvement This programme underwent an extensive evaluation which focused on the cultural changes, the changes in discourse and the clinical outcomes. We will be reporting on the final evaluation with a focus on how the programme has changed the way teams interact and how this could have an impact on clinical outcomes.

Effects of changes We will report a change in the way teams interacted, a change in the language they used, and a change in some clinical outcomes. We will also provide reflections on how to spread and sustain such initiatives.

PLEASE NOTE some aspects of this project have been published; the presentation will refer to these, offering a different perspective. 